\title{
Validation of devices for characterization of hybrid 3D printed embroidery TENG for energy harvesting
}

\author{
Hasan Riaz Tahir ${ }^{1, *}$, Benny Malengier ${ }^{1}$, Carla Hertleer ${ }^{1}$, Lieva Van Langenhove ${ }^{1}$ \\ ${ }^{1}$ Centre for Textile Science and Engineering, Department of Materials, Textiles and Chemical Engineering, Ghent \\ University, 9000 Gent, Belgium \\ *Corresponding author E-mail address: hasan.tahir@ugent.be
}

\section{INFO}

CDAPT, ISSN 2701-939X

Peer reviewed article

2022, Vol. 3, No. 1, pp. 1-8

DOI 10.25367/cdatp.2022.3.p1-8

Received: 16 December 2022

Accepted: 10 February 2022

Available online: 01 March 2022

\section{Keywords}

hybrid nanogenerator,

flexible filament,

3D printing,

\section{embroidery,}

tapping characterization, conductive multifilament, hybrid yarn

\begin{abstract}
A textile-based triboelectric nanogenerator (TENG) is an energy harvesting flexible and lightweight device that converts mechanical energy to electrical energy. This work presents characterization of a novel hybrid 3D printed embroidery TENG for energy harvesting. The digital embroidery part is done on Brother Embroidery Machine PR670E with polyester multifilament conductive hybrid thread (CleverTex) with a linear thread resistance of $280 \Omega / m$. This embroidery thread is fully compatible with the standard textile embroidery process. The thread is highly suitable for embroidery due to its very good mechanical properties and no loop formation during embroidery. These features make the thread especially suitable for high production quality. It could be used as needle thread or bobbin thread. For the preparation of the embroidery part, the polyester multifilament conductive hybrid thread is used as needle thread with $100 \%$ polyester Madeira thread as bobbin thread. These threads have non-toxic, non-skin irritation properties, which makes them suitable for smart wearable energy harvesting applications. Furthermore, these threads are coated with silicone-paraffin emulsions that improve their running during the embroidery process. Among the possible stitch types (satin, fill, prog. fill, piping, motif, cross, concentric circle, radial, spiral, flexible spiral, stippling, net fill, zigzag net fill, and decorative fill), fill stitch with medium stitch density and 4.5 lines per $\mathrm{mm}$ has been used to develop this energy harvesting sample. The $3 D$ printed textile fabric is prepared with extremely flexible filament with a tensile elongation at break of $1400 \%$. The output voltage is $200 \mathrm{~V}$ and $103 \mathrm{~V}$ for tapping and friction characterization, respectively.
\end{abstract}

(C) 2022 The authors. Published by CDAPT.

This is an open access article under the CC BY-NC-ND license https://creativecommons.org/licenses/ peer-review under responsibility of the scientific committee of the CDAPT.

(c) 2022 CDAPT. All rights reserved. 


\section{Introduction}

Triboelectric nanogenerators (TENGs) couple triboelectric effect and electrostatic induction to convert mechanical energy into electricity; because of the benefit of fabrication, tremendous progress has been created since the first discovery in 2012 [1,2]. The triboelectric effect is everywhere in our daily life and results from two different materials coming into contact. Sometimes, it is considered an adverse consequence due to a spark near the electronics parts [3], but these generated charges could also be collected to harvest energy [4-6]. The triboelectric nanogenerator is one of the promising candidates for powering wearable electronics with flexibility, lightweight and good performance $[7,8]$. There are mainly two categories of TENG, i.e. fiber-based TENG and fabric-based TENG [9, 10]. Materials that are used to develop (TENGs) have received special consideration for wearable applications. TENGs are also used in combination with other energy harvesting materials [2,11]. A triboelectric nanogenerator was reported to be made with embroidery on the textile substrate using the pile embroidery in order to have a rough and high surface area to get a high result voltage of $113 \mathrm{~V}$ [12]. There is an investigation concerning the result of textile process technique (weaving, knitting, sewing, embroidery, etc.) and structure pattern on the TENG's output performance [13].

There are different modes of triboelectric nanogenerators that are used to harvest energy, offering a variety of choices of application areas on harvesting energy from human motion [14-16]. There are devices having different options to characterize the electrostatic or triboelectric behavior of textile materials [17-19]. On a fundamental level, any material with a particular charge density can be utilized to develop a TENG, which brings about a broad scope of materials at the furthest edges of the triboelectric series [20-22]. A 3-dimensional ultra-flexible TENG was reported to harvest biomechanical energy [23]. The all-printed triboelectric nanogenerator (AP-TENG) is a combination of the advantages of 2D and 3D printing innovations. The primary edge is framed by 3D printing as a center shell structure, which successfully changes over outside vibrations into consistent sliding movement. An AP-TENG reported in the literature produces a high momentary voltage of $98.2 \mathrm{~V}$ [22]. Triboelectric generators and sensors have large potential as self-controlled wearable gadgets for energy collecting biomechanical motions. There are four different basic modes of triboelectric nanogenerators; vertical contact separation mode, single electrode mode; contact sliding mode, and freestanding mode, as shown in Fig. 1. These modes are also applicable for tapping and sliding devices. The vertical contact separation mode and single electrode mode are for the tapping motion, and contact sliding and freestanding mode are for the sliding device.
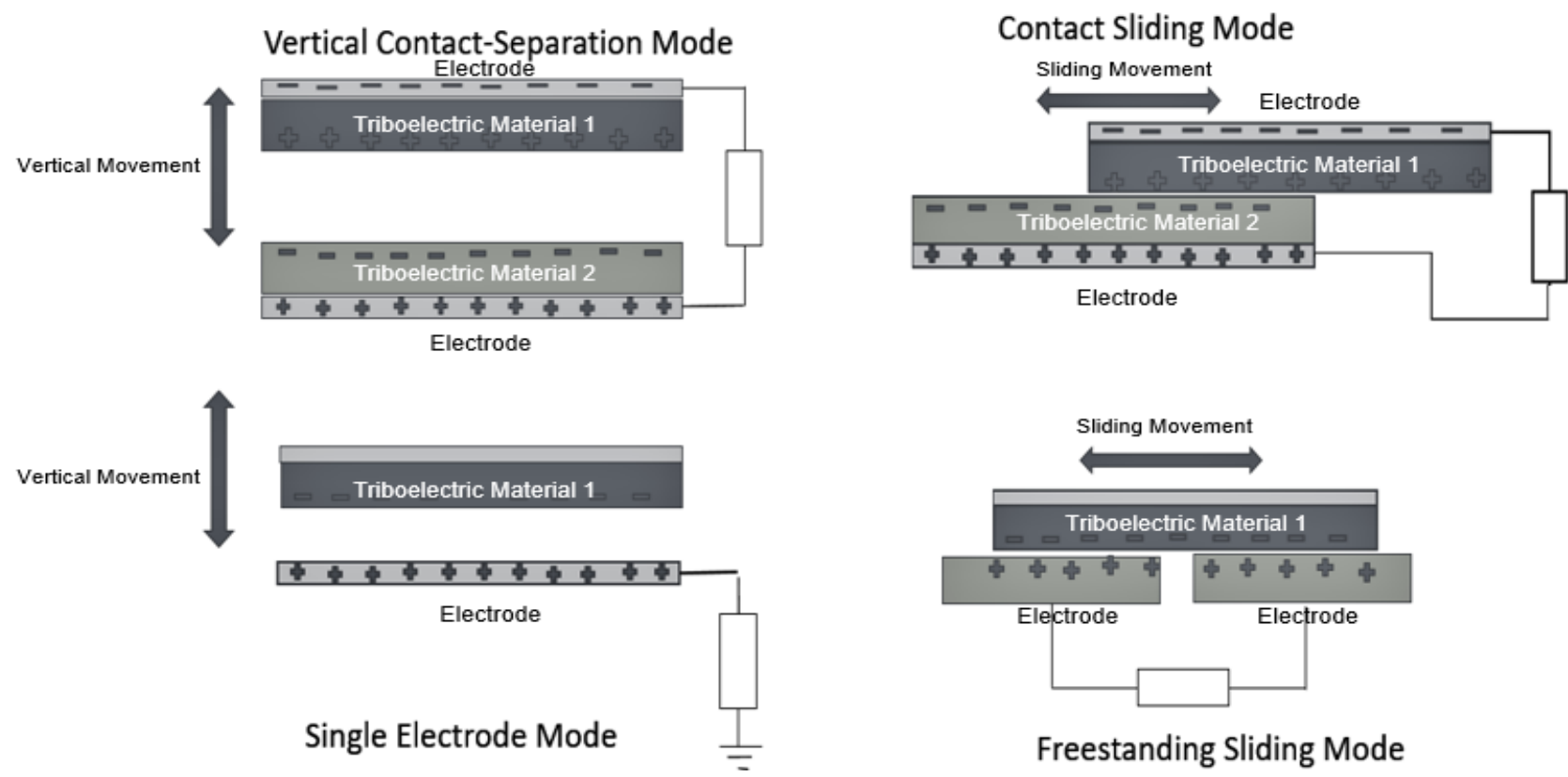

Fig. 1 Four fundamental modes of triboelectric characterization [17]. 
In this study, we use both generation modes of triboelectric charges to characterize the samples. We develop hybrid materials and a technology-based triboelectric nanogenerator. We use the commercially available yarn with a commercially available embroidery machine, which is suitable for making an exciting fabric design and harvesting energy. 3D printing filament that is used for triboelectric generator is good material for both creating the insole and making print on the textile substrate, so it is very useful for harvesting energy not only from sliding motion if it is attached to wearables, but it could also harvest energy from tapping motion if it is used as an insole.

\section{Materials and methods}

This TENG has been made with an embroidery machine and 3D printing on a textile substrate. The embroidery machine PR 670E has been used to develop the embroidered sample. This machine has six needle heads and can sew different embroidery patterns. There are different stitch types, e.g., satin, fill, prog. fill, piping, motif, cross, concentric circle, radial, spiral, flexible, stippling, net fill, zigzag net fill, zigzag, and decorative fill stitch.

The sample for the energy harvesting was made with polyester multifilament conductive hybrid thread as needle thread and $100 \%$ polyester Madeira yarn as bobbin thread with fill stitch. The manufacturer of the conductive yarn is CLEVERTEX®. For the upper side of the sample, the stitch length was $2 \mathrm{~mm}$ with a line density of 4.5 lines per $\mathrm{mm}$. The line density was medium for the under sewing with 4.5 lines per $\mathrm{mm}$ at a $45^{\circ}$ angle. The sample was prepared at 700 rounds per minute, showing the good strength and running of the stitching thread used for the embroidery. Fig. 2 shows the embroidery attributes.

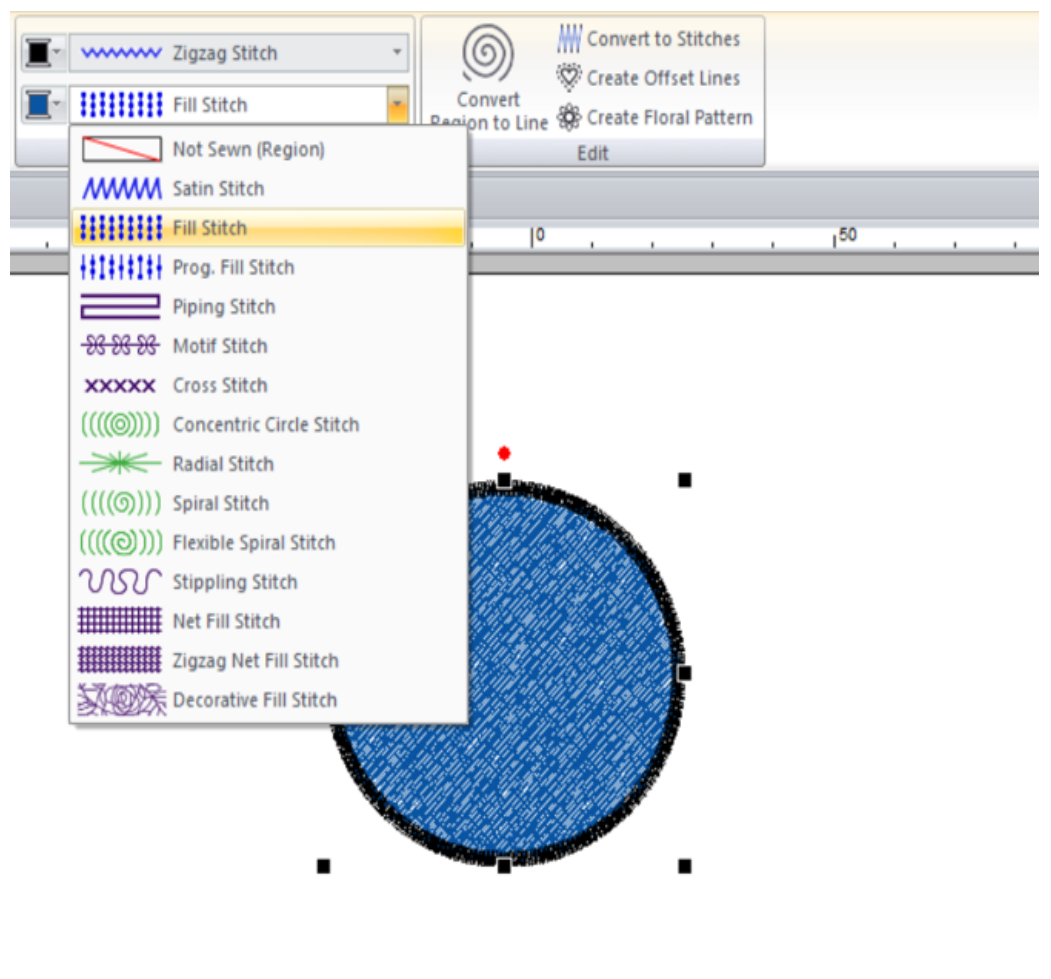

(a)

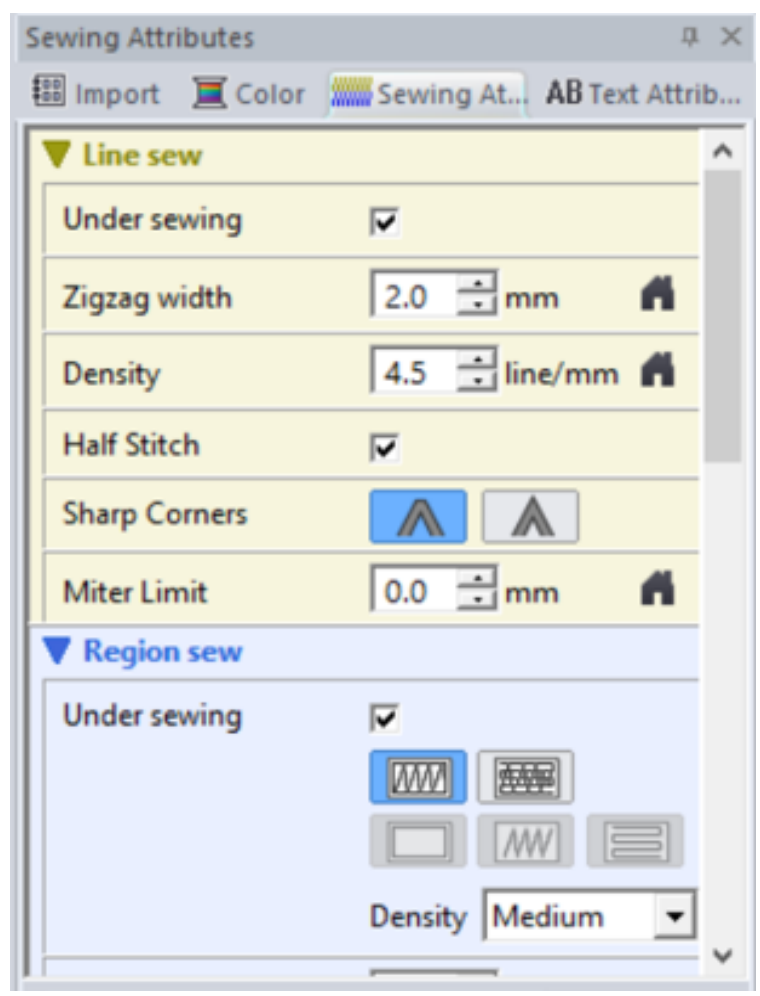

(b)

Fig. 2 Cont. 


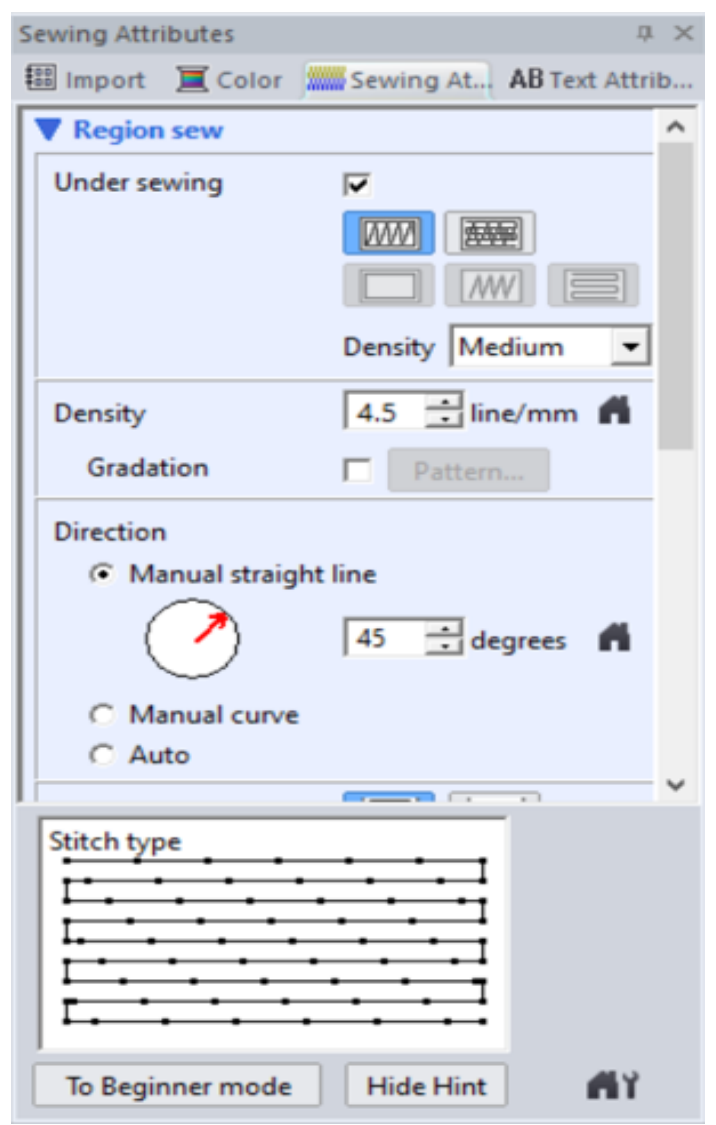

(c)

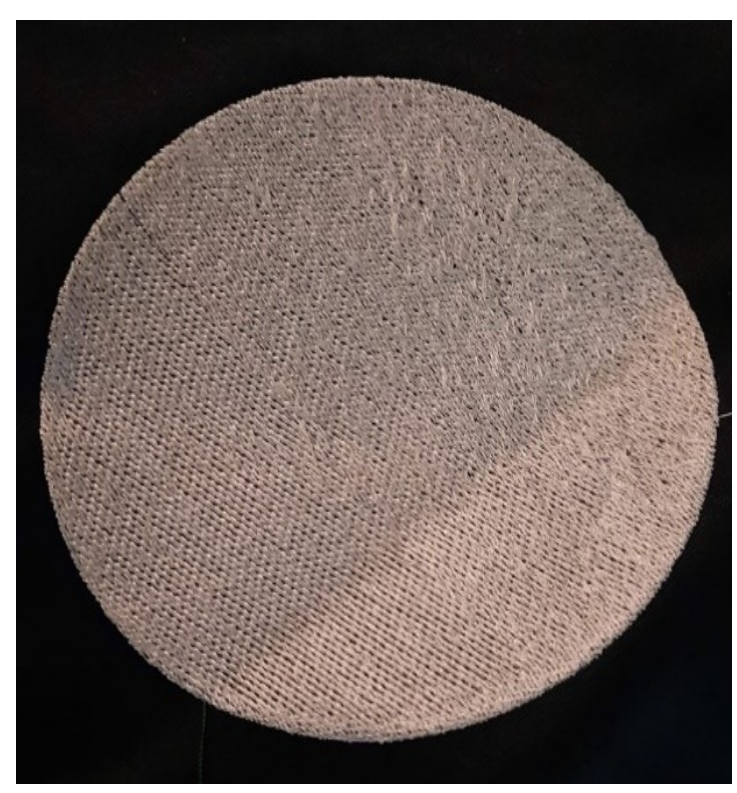

(d)

Fig. 2 (a) Embroidery attributes - selection of stitch type; (b) sewing attributes - sewing stitch length of $2 \mathrm{~mm}$, stitch density medium with 4.5 lines per $\mathrm{mm}$; (c) under sewing attributes - medium stitch density, 4.5 lines per $\mathrm{mm}$ at an angle of 45 degrees; (d) embroidered sample with conductive yarn to harvest energy.

3D printing on the fabric is performed with Filaflex, a high flexible and elastic material with a tensile elongation at break of $1400 \%$. Flexible filament printing is possible at lower temperatures $\left(215-225^{\circ} \mathrm{C}\right)$, and the filament must be moisture-free. The flexible filament also has excellent print bed adhesion, so they do not require a heated print bed, tape, lacquer, or spray adhesive. In addition, it is odorless and resistant to solvents such as acetone or petrol. Since it is a non-toxic material, it can also contact the skin.

The filament can be used for insoles, especially orthotic insoles. Another application of this flexible filament is textile parts and accessories: clothing, textile fabrics, prints on garments, bags, etc. The printing speed required is $20-30 \mathrm{~mm} / \mathrm{s}$ with an optimal layer height of $0.2 \mathrm{~mm}$. It has a remarkable hydrolysis resistance, high resistance to bacteria, and low-temperature flexibility properties.

Fig. 3 shows the 3D printing on the textile substrate. Two different devices were used to characterize the samples for the tapping and sliding characterization at a frequency of $2 \mathrm{~Hz}$.

For the tapping device, the dwell time, contact time, and up was $200 \mathrm{~ms}, 250 \mathrm{~ms}, 50 \mathrm{~ms}$, and for the sliding device, a frequency of $2 \mathrm{~Hz}$ is controlled with a variable power supply (24 V required for $2 \mathrm{~Hz}$ ). Fig. 4 shows the energy harvesting TENG with characterization devices for tapping and lateral sliding characterization. 


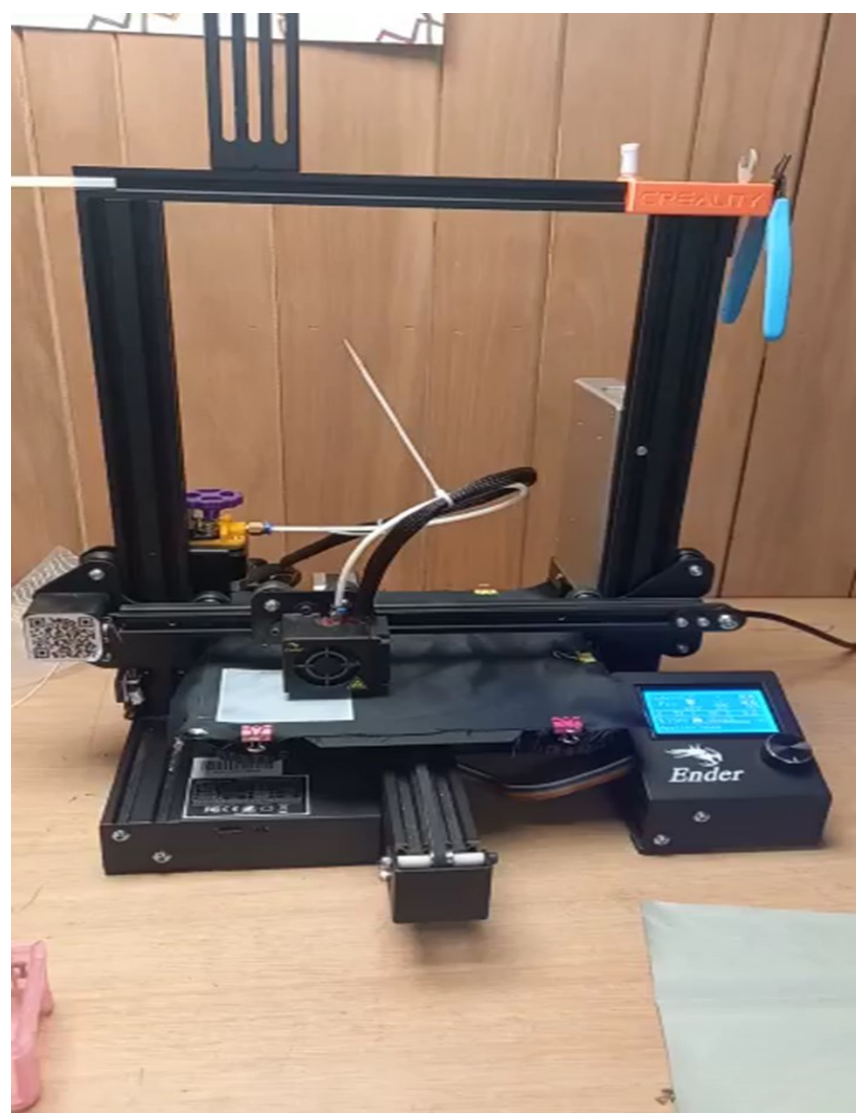

(a)

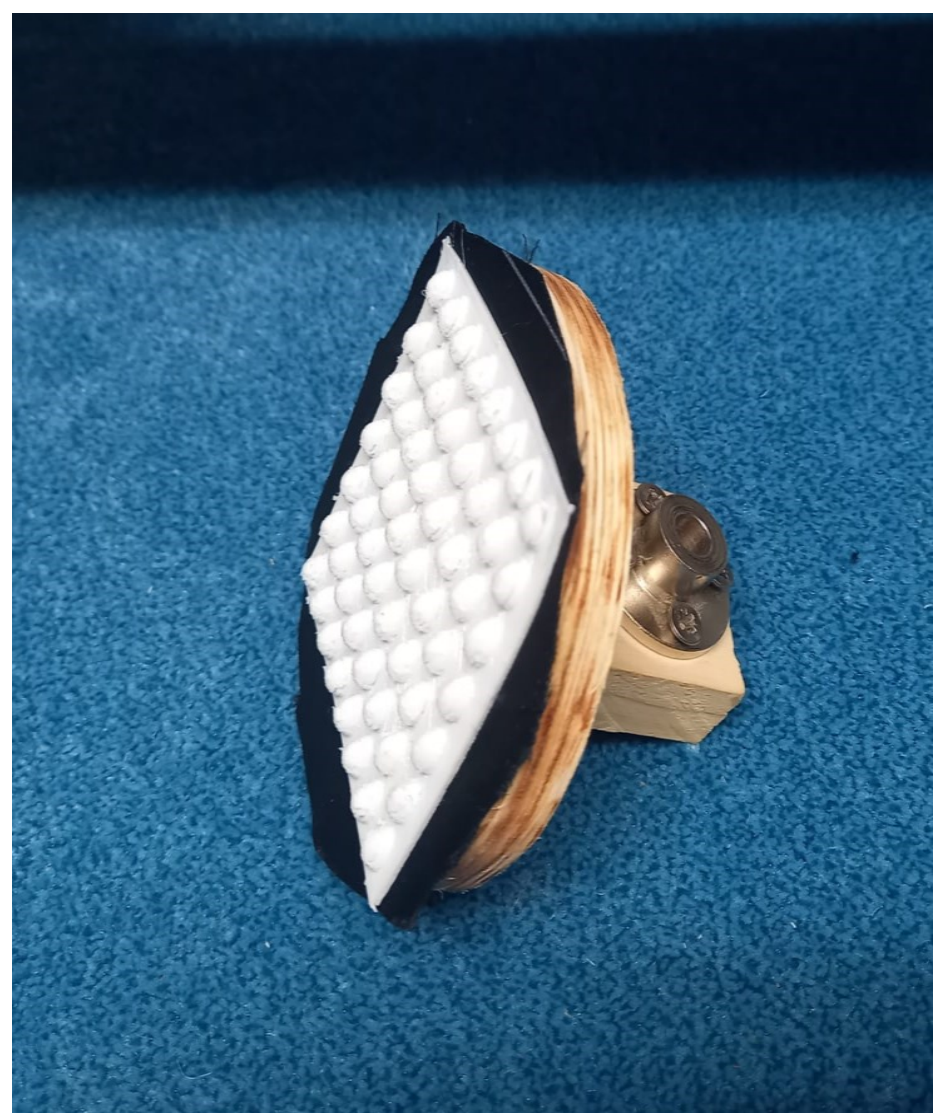

(b)

Fig. $33 D$ printing on the textile substrate. (a) Filaflex is used to print on the textile substrate with flexible and elastic materials with a tensile elongation at break of $1400 \%$ (b) flexible, lightweight and soft 3D printed sample.

Low Voltage Processing Unit National Instrument DAQ

Pressure valve Unit

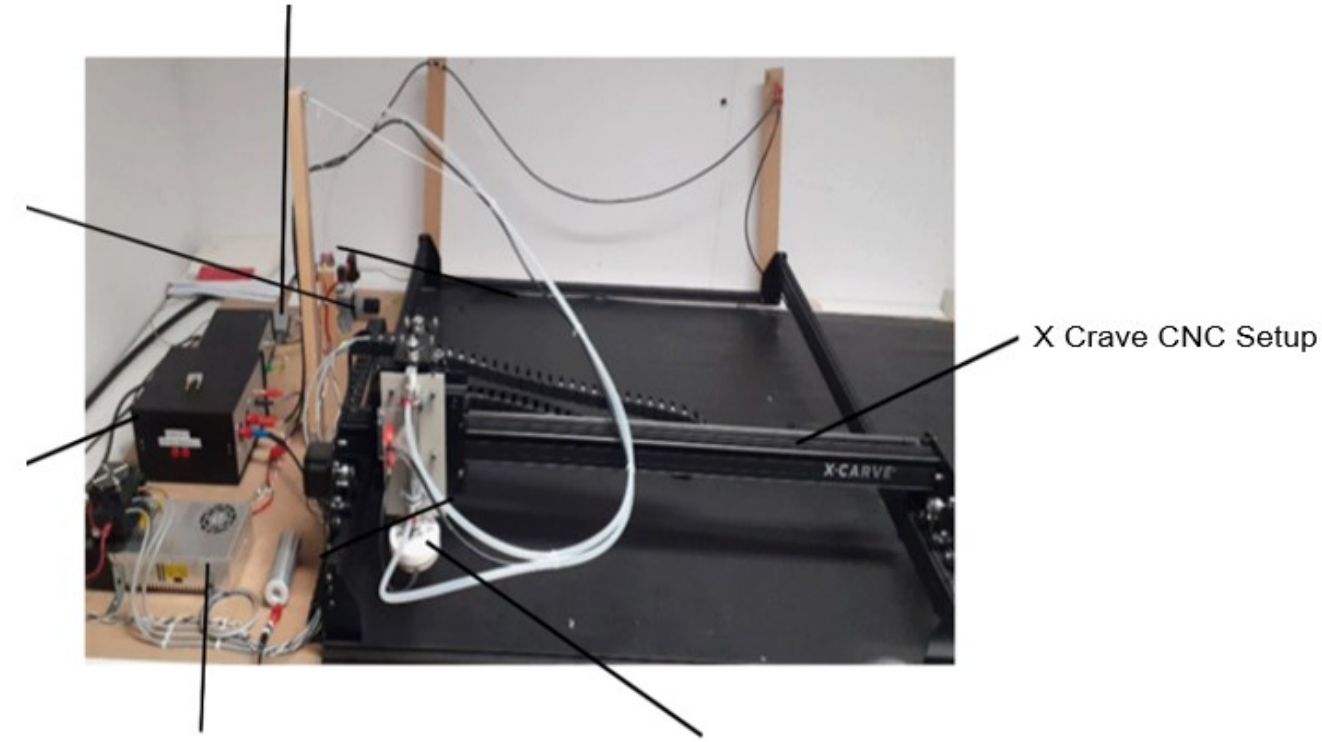

Power Source and Rasberry Pi to process CNC Command
Automated Contact

Separation Part

(a)

Fig. 4 Cont. 


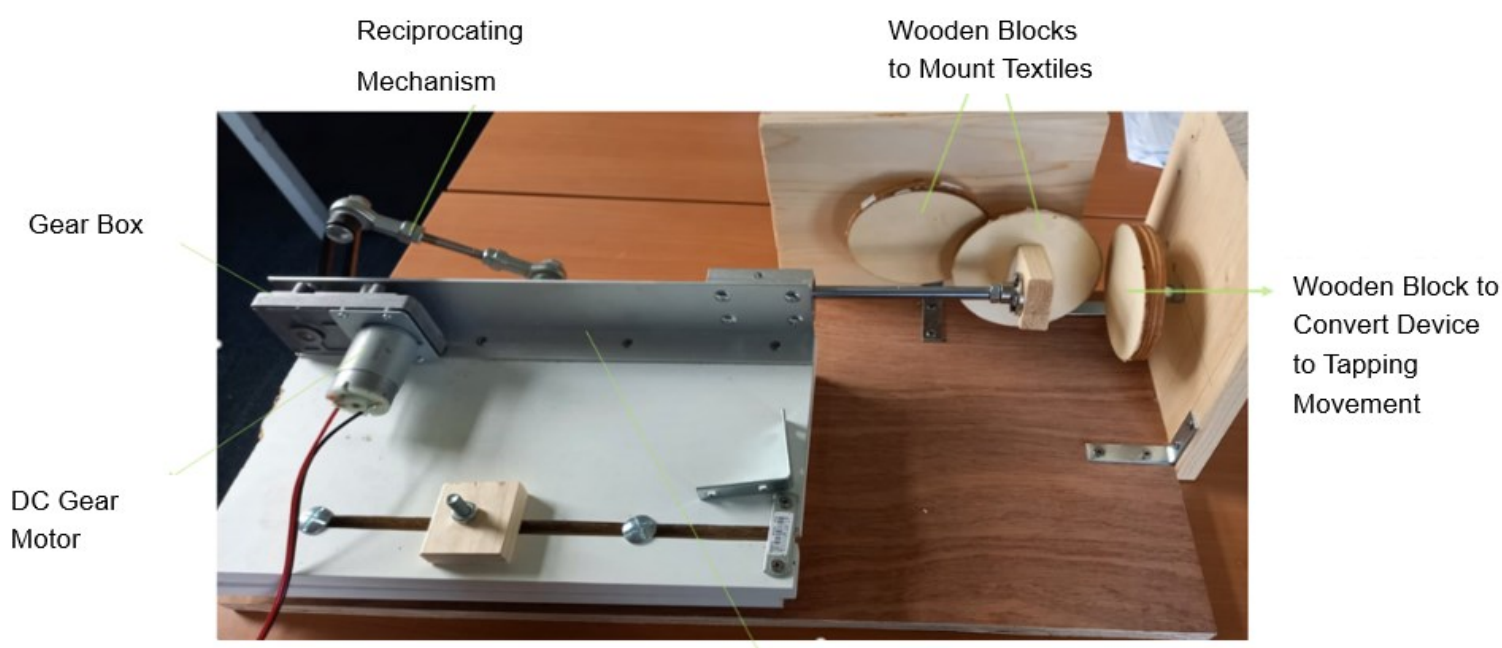

Aluminum Frame to Fix Motor and

Reciprocating Mechanism

(b)

Fig. 4 Energy harvesting TENG with characterization devices: (a) Tapping characterization device for TENG; (b) lateral sliding characterization device for TENG.

\section{Results and discussion}

Fig. 4 shows the TENG's output voltage of the tapping and sliding mode. For the tapping characterization, the generated voltage is increased over time up to $200 \mathrm{~V}$. The overall voltage waveform is in negative direction due to dominating negative triboelectric behavior of $3 \mathrm{D}$ printing filament. In contrast, for the sliding device, the slope of the voltage vs. time is not much steeper than in tapping mode, with a generated maximum of $98 \mathrm{~V}$. The measured output power of the TENG is $400 \mathrm{~mW}$ and $96 \mathrm{~mW}$ at the resistance of $100 \mathrm{k} \Omega$ resistance, respectively. The output power for the tapping mode is higher, reflecting the printed dot-structure to respond more to pressing than to sliding.

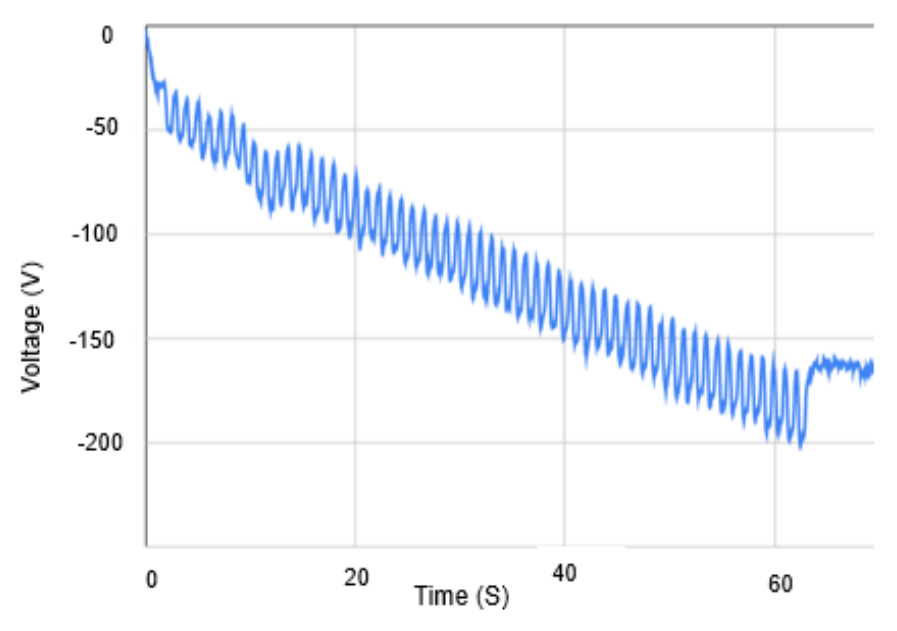

(a)

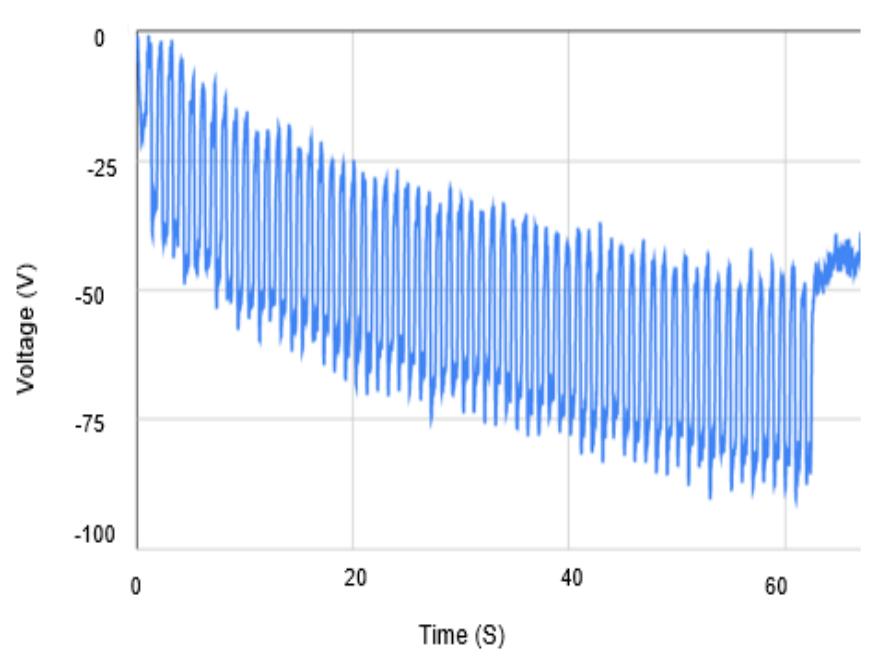

(b)

Fig. 5 Output voltages of the tapping and sliding mode of TENG: (a) tapping characterization voltage versus time; (b) lateral sliding characterization voltage versus time.

\section{Conclusions}

We presented a hybrid TENG, developed from a conductive polyester multifilament conductive hybrid thread (CleverTex) and $3 \mathrm{D}$ printing of highly flexible and elastic material with a tensile elongation at 
break of $1400 \%$. Both triboelectric fabrics have good flexibility and softness after the embroidery and printing. The triboelectric performance measured on tapping and friction devices at a frequency of $2 \mathrm{~Hz}$ gave output voltages of $200 \mathrm{~V}$ and $98 \mathrm{~V}$, and the measured output power was $400 \mathrm{~mW}$ and $96 \mathrm{~mW}$ at a resistance of $100 \mathrm{k} \Omega$, respectively.

\section{Author Contributions}

Author contributions statement: H. R. Tahir: conceptualization, methodology, validation, writing - original draft preparation; B. Malengier: conceptualization, methodology, software, supervision; C. Hertleer: visualization, project administration; L. Van Langenhove: Software, supervision, project administration, funding acquisition. All authors have read and agreed to the published version of the manuscript.

\section{Funding:}

B. M. and L. V. L. were funded by the ICT-Tex project EU project (Nr. 612248-EPP-1-2019-1BGEPPKA2-KA); H. R. T. by HEC (Higher Education Commission), Pakistan: HRDI-UESTP Scholarship Project.

\section{Acknowledgements}

Thanks to the Ingegno Maker Space, Drongen team, Belgium, for using their digital embroidery machine and 3D printer equipment to develop TENG energy harvesting samples.

\section{Conflicts of Interest}

The authors declare no conflict of interest.

\section{References}

1. Fan, F.-R.; Tian, Z.-Q.; Wang, Z. L. Flexible triboelectric generator. Nano Energy 2012, 1 (2), 328-334. DOI: https://doi.org/10.1016/j.nanoen.2012.01.004.

2. Wu, C. S.; Wang, A. C.; Ding, W. B.; Guo, H. Y.; Wang, Z. L. Triboelectric nanogenerator: a foundation of the energy for the new era. Advanced Energy Materials 2019. 9 (1), 1802906. DOI: https://doi.org/10.1002/aenm.201802906.

3. Smallwood, J. Reducing static electricity in carpets, in Advances in carpet manufacture; Elsevier, 2018; pp. 135-162. DOI: https://doi.org/10.1016/B978-0-08-101131-7.00008-3.

4. Tani, H.; Sugimoto, M.; Fushihara, K.; Nakao, Y.; Renguo, R.; Koganezawa, S.; Tagawa. Energy harvesting from triboelectric nanogenerator attached inside folling tire. In 2019 19th International Conference on Micro and Nanotechnology for Power Generation and Energy Conversion Applications (PowerMEMS). 2019. DOI: https://doi.org/10.1109/PowerMEMS49317.2019.002.

5. Wang, Z. L.; Jiang, T.; Xu, L. Toward the blue energy dream by triboelectric nanogenerator networks. Nano Energy 2017, 39, 9-23. DOI: https://doi.org/10.1016/j.nanoen.2017.06.035.

6. Wang, Z. L. Triboelectric nanogenerator (TENG) - sparking an energy and sensor revolution. Advanced Energy Materials 2020. 10 (17), 2000137. DOI: https://doi.org/10.1002/aenm.202000137.

7. Bera, B. Literature review on triboelectric nanogenerator. Imperial Journal of Interdisciplinary Research (IJIR) 2016, 2 (10), 1263-1271.

8. Repoulias, A.; Vassiliadis, S.; Galata, S. F. Triboelectricity and textile structures. The Journal of The Textile Institute 2021, 112 (10), 1580-1587. DOI: https://doi.org/10.1080/00405000.2020.1829331.

9. Paosangthong, W.; Torah, R.; Beeby, S. Recent progress on textile-based triboelectric nanogenerators. Nano Energy 2019, 55, 401-423. DOI: https://doi.org/10.1016/j.nanoen.2018.10.036.

10. Rathore, S.; Sharma, S.; Swain, B. P.; Ghadai, R. Kr. A critical review on triboelectric nanogenerator. IOP Conf. Ser.: Mater. Sci. Eng. 2018, 377, 012186.

11. Jung, W.-S.; Kang, M.-G.; Moon, H. G.; Baek, S-H.; Yoon, S.-J.; Wang, Z.-L.; Kim, S.-W.; Kang, C.-Y. High output piezo/triboelectric hybrid generator. Scientific Reports 2015. 5 (1), 9309. DOI: https://doi.org/10.1038/srep09309

12. Pyo, S.; Kim, M.-Ol; Kwon, D.-S.; Kim, W. D.; Yang, J.-H.; Cho, H. S.; Lee, J. H.; Kim, J. B. All-textile wearable triboelectric nanogenerator using pile-embroidered fibers for enhancing output power. Smart Materials and Structures 2020. 29 (5), 055026. DOI: https://doi.org/10.1088/1361-665X/ab710a. 
13. Cui, N. Y.; Gu, L.; Lei, Y. M.; Liu, J. M.; Qin, Y.; Ma, X. H.; Hao, Y.; Wang, Z. L. Dynamic behavior of the triboelectric charges and structural optimization of the friction layer for a triboelectric nanogenerator. ACS Nano 2016, 10 (6), 6131-6138. DOI: https://doi.org/10.1021/acsnano.6b02076.

14. Xia, K. Q.; Zhu, Z. Y.; Zhang, H. Z.; Du, C. L.; Xu, Z. W.; Wang, R. J. Painting a high-output triboelectric nanogenerator on paper for harvesting energy from human body motion. Nano Energy 2018, 50, 571-580. DOI: https://doi.org/10.1016/j.nanoen.2018.06.019.

15. Zhou, T., Zhang, C.; Han, C. B.; Fan, F. R.; Tang, W.; Wang, Z. L. Woven structured triboelectric nanogenerator for wearable devices. ACS Applied Materials \& Interfaces 2014, 6 (16), 14695-14701. DOI: https://doi.org/10.1021/am504110u.

16. Xing, F.; Jie, Y.; Cao, X.; Li, T.; Wang, N. Natural triboelectric nanogenerator based on soles for harvesting low-frequency walking energy. Nano Energy 2017, 42, 138-142. DOI: https://doi.org/10.1016/j.nanoen.2017.10.029.

17. Tahir, H. R.; Malengier, B.; van Daele, D.; van Langenhove, L. Validation of a platform for the electrostatic characterization of textile. Electronics 2022, 11 (1), 115. DOI: https://doi.org/10.3390/electronics11010115.

18. Kim, W.-G.; Kim, D.-W.; Tcho, I.-W.; Kim, J.-K.; Kim, M.-S.; Choi, Y.-K. Triboelectric nanogenerator: Structure, mechanism, and applications. ACS Nano 2021, $15 \quad$ (1), 258-287. DOI: https://doi.org/10.1021/acsnano.0c09803.

19. Zhu, G.; Peng, B.; Chen, J.; Jing, Q. S.; Wang, Z. L. Triboelectric nanogenerators as a new energy technology: From fundamentals, devices, to applications. Nano Energy 2015, 14, $126-138$. DOI: https://doi.org/10.1016/j.nanoen.2014.11.050.

20. Seol, M.; Kim, S. S.; Cho, Y. C.; Byun, K.-E.; Kim, H. Y.; Kim, J. H.; Kim, S. K.; Kim, S.-W.; Shin, H.-J.; Park, S. J. Triboelectric series of 2D layered materials. Advanced Materials 2018, 30 (39), 1801210. DOI: https://doi.org/10.1002/adma.201801210.

21. Zou, H. Y.; Zhang, Y.; Guo, L. T.; Wang, P. H.; He, X.; Dai, G. Z.; Zheng, H. W.; Chen, C. Y.; Wang, A. C.; Xu, C.; Wang, Z. L. Quantifying the triboelectric series. Nature Communications 2019, 10 (1), 1427. DOI: https://doi.org/10.1038/s41467-019-09461-x.

22. Zou, H. Y.; Guo, L. T.; Xue, H.; Zhang, Y.; Shen, X. F.; Liu, X. T.; Wang, P. H.; He, X.; Dai, G. Z.; Jiang, P.; Zheng, H. W.; Zhang, B. B.; Xu, C.; Wang, Z. L. Quantifying and understanding the triboelectric series of inorganic non-metallic materials. Nature Communications 2020, 11 (1), $2093 . \quad$ DOI: https://doi.org/10.1038/s41467-020-15926-1

23. Chen, B. D.; Tang, W.; Jiang, T.; Zhu, L. P.; Chen, X. G.; He, C.; Xu, L.; Guo, H. G.; Lin, P.; Li, D.; Shao, J. J.; Wang, Z. L. Three-dimensional ultraflexible triboelectric nanogenerator made by 3D printing. Nano Energy 2018, 45, 380-389. DOI: https://doi.org/10.1016/j.nanoen.2017.12.049. 